\title{
Afamin and adropin in patients with alcohol-induced liver cirrhosis
}

Andrzej Prystupa ${ }^{1}$, Paweł Kiciński ${ }^{2}$, Dorota Luchowska-Kocot ${ }^{3}$, Jarosław $\mathrm{Sak}^{4}$, Tomasz Prystupa $^{5}$, Ko-Hsin Chen ${ }^{6}$, Yu-Chieh Chen ${ }^{6}$, Lech Panasiuk ${ }^{7}$, Wojciech Załuska ${ }^{8}$

${ }^{1}$ Department of Internal Diseases, Medical University of Lublin, Lublin, Poland

${ }^{2}$ Department of Experimental Hematooncology, Medical University of Lublin, Lublin, Poland ${ }^{3}$ Department of Medical Chemistry, Medical University of Lublin, Lublin, Poland

${ }^{4}$ Department of Ethics and Human Philosophy, Medical University of Lublin, Lublin, Poland ${ }^{5}$ Independent Public Teaching Hospital No 4 in Lublin, Poland

${ }^{6}$ Students' Scientific Society, Medical University of Lublin, Lublin, Poland

${ }^{7}$ Department of Internal Diseases and Hypertension, Institute of Rural Medicine, Lublin, Poland

${ }^{8}$ Department of Nephrology, Medical University of Lublin, Lublin, Poland

\section{Corresponding author:}




\begin{abstract}
Introduction: Liver cirrhosis develops in about $10 \%$ of alcohol abusers. To date, a number of cells and cytokines have been identified, which are involved in induction of liver fibrotic processes. Nevertheless, the pathogenesis of liver cirrhosis has not been fully elucidated.

The aim of the present study was to determine serum concentrations of afamin and adropin in patients with alcoholic liver cirrhosis and to define their correlation with the stage of disease.
\end{abstract}

Materials and methods: The study included 99 patients with alcoholic cirrhosis from the region of Lublin, (Eastern Poland). Liver cirrhosis was diagnosed based on clinical features, history of heavy alcohol consumption, laboratory tests and abdominal ultrasonography. The control group consisted of 20 healthy individuals without liver disease who did not abuse alcohol. The serum afamin and adropin concentrations were determined using ELISA kits.

Results: The concentration of afamin was found to be significantly lower in patients with compensated alcoholic liver cirrhosis, i.e. P-Ch B $(85.1 \pm 40.6 \mu \mathrm{g} / \mathrm{ml})$ and P-Ch C $(56.4 \pm 32.3$ $\mu \mathrm{g} / \mathrm{ml})$ individuals, as compared to the control group $(135.9 \pm 43.6 \mu \mathrm{g} / \mathrm{ml})$; p-value was $<0.01$ and $<0.001$, respectively. As far as adropin is concerned, a reverse relationship was demonstrated: the highest concentration was found in patients with P-Ch C $(11.7 \pm 5.7 \mathrm{ng} / \mathrm{ml})$ cirrhosis. Furthermore, the above concentration was significantly higher compared to patients with P-Ch A cirrhosis $(7.2 \pm 2.8 \mathrm{ng} / \mathrm{ml} ; \mathrm{p}<0.05)$ and controls $(7.5 \pm 2.6 \mathrm{ng} / \mathrm{ml} ; \mathrm{p}<0.05)$.

Conclusions: The concentration of afamin decreases with the severity of alcoholic liver cirrhosis, which most likely results from impaired hepatic synthesis. Otherwise, the higher the stage of disease according to the Child-Pugh score, the higher the concentration of adropin. 


\section{Introduction}

Chronic alcohol abuse can lead to the development of alcoholic liver disease, chronic pancreatitis and many other ailments. Liver cirrhosis is a relatively common disease; its incidence is estimated at 200-300 per 100000 individuals. In Poland, the liver cirrhosisassociated mortality increased from 5.14/100 000 in 1980 to $7.6 / 100000$ inhabitants in $2010^{\mathrm{i}}$. Three forms of alcoholic livers cirrhosis are distinguished: alcoholic fatty liver, acute alcoholic hepatitis and liver cirrhosis. The factors increasing the risk of alcoholic liver cirrhosis include: the duration of excessive alcohol consumption, female gender, consumption of high-proof alcohol and coexistence of other liver diseases.

Liver cirrhosis develops in about $10 \%$ of alcohol abusers. Acetaldehyde is the main metabolite of ethyl alcohol resulting in liver damage. The major process leading to cirrhosis is fibrosis which is associated with imbalance between fibrogenesis and fibrolysis ${ }^{\text {ii }}$. To date, a number of cells and cytokines have been identified, which are involved in induction of liver fibrotic processes ${ }^{\text {iii }}$. Nevertheless, the pathogenesis of liver cirrhosis has not been fully elucidated. Recent studies have demonstrated that metabolic disorders are likely to affect the development of liver cirrhosis ${ }^{\mathrm{iv}}$.

Afamin and adropin have recently been found to be useful markers of the metabolic syndrome $^{\mathrm{v}}$. Their concentrations have not as yet been determined in patients with alcoholic liver cirrhosis. Afamin is a plasma glycoprotein bounding vitamin E produced mainly in the liver. The most recent studies have shown that plasma concentrations of afamin are strongly correlated with metabolic syndrome indices ${ }^{\mathrm{vi}}$. Adropin is a peptide hormone involved in energy homeostasis and regulation of metabolism of glucose and fatty acids ${ }^{\mathrm{vii}}$. 
The aim of the present study was to determine serum concentrations of afamin and adropin in patients with alcoholic liver cirrhosis and to define their correlation with the stage of disease.

\section{Material and methods}

\section{Patients}

The study included 99 patients with alcoholic cirrhosis from the region of Lublin, (Eastern Poland). Liver cirrhosis was diagnosed based on clinical features, history of heavy alcohol consumption, laboratory tests and abdominal ultrasonography. The patients with alcoholic hepatitis, hepatocellular carcinoma, viral and autoimmune diseases were excluded from the study. Other exclusions criteria were: type 2 diabetes, obesity, acute infections (e.g., pneumonia, spontaneous bacterial peritonitis), acute and chronic heart failure (> NYHA I), acute and chronic respiratory disorders resulting in respiratory insufficiency, acute kidney injury and chronic kidney disease ( $>$ stage G2). Both clinical assessment and laboratory tests were used to exclude the underlying liver diseases in the control group. The degree of liver cirrhosis was evaluated according to the Pugh-Turcotte-Child criteria (Pugh-Child score) ${ }^{\text {viii }}$. Based on them patients were assigned to one of three groups: Pugh-Child (P-Ch) A - 29 with stage A, P-Ch B - 26 with stage B and P-Ch C - 34 with stage C of liver cirrhosis. The control group consisted of 20 healthy individuals without liver disease who did not abuse alcohol. There were no significant age- or gender-related differences in the groups (Table 1). Detailed demographic, clinical and biochemical characteristics of patients are presented in Table 1 and 2 .

The study protocol was approved by the Ethics Committee. All subjects gave their written informed consent for participation in the study. 


\section{Determination of serum afamin concentration}

The afamin concentration was determined using human afamin ELISA kit (BioVendor, Czech Republic) according to the manufacture's procedure. After the addition of the appropriately diluted samples (100-fold diluted serum), standards and water (blank sample), 60-minute incubation was provided. During incubation, human afamin was bounded to antibodies absorbed to the microwells. After incubation and washing, monoclonal antihuman afamin antibody conjugated with horseradish peroxidase (HRP) was added to the wells and incubated for 60 minutes with captured afamin. After a thorough wash, the remaining conjugate was allowed to react with the substrate solution (TMB). The reaction was stopped by addition of acidic solution. Absorbance of coloured products was measured spectrophotometrically $(\lambda=450 \mathrm{~nm})$ and their concentration was determined using a standard curve prepared for standards. The results were multiplied by a dilution factor (100).

\section{Determination of serum adropin concentration}

Adropin concentration was determined using sandwich enzyme immunoassay kit for adropin (Cloud Clone Corp., Katy, TX, USA) according to the manufacturer's procedure. Samples (20-fold diluted serum), standards and water (blank sample) were applied onto a microtiter plate pre-coated with an antibody specific to adropin. After 60 minutes of incubation the content of the wells was removed, the plate was washed and a biotinconjugated antibody specific to adropin was added. Next, Avidin conjugated to horseradish peroxidase (HRP) was added to each microplate well and incubated. During the next step, TMB substrate solution was added to each well. In the wells containing adropin, biotinconjugated antibody and enzyme-conjugated Avidin exhibited a colour change. The enzymesubstrate reaction was terminated by addition of sulphuric acid solution and the absorbance of standards and samples was measured spectrophotometrically at a wavelength $450 \mathrm{~nm}$. The 
concentration of adropin in the samples was determined using a standard curve constructed for standards. The results were multiplied by a dilution factor (20).

\section{Statistical analysis}

STATISTICA 13 (Statsoft, Inc.) was used for data analysis. Continuous variables were expressed as the mean \pm standard deviation (SD). Before calculations, variables were checked for normality using the Shapiro-Wilk test; the Brown-Forsythe`s test was applied to test equality of variances. To compare the results between more than two groups, one-way ANOVA test was used. The Tukey`s test was applied for detailed identification of statistically different groups. Correlations among variables were performed using the Pearson's correlation test. Stepwise multiple linear regression analysis was applied to determine the potential independent influence of various factors on afamin and adropin concentrations. Qualitative variables are shown as indicators of structure (percentage); for intergroup comparisons the $\chi^{2}$ test was used. Receiver-operators characteristic (ROC) curves were used to assess diagnostic accuracy of afamin and adropin. For all tests, $\mathrm{p}<0.05$ was considered as statistically significant.

\section{Results}

The concentration of afamin was found to be significantly lower in patients with compensated alcoholic liver cirrhosis, i.e. P-Ch B $(85.1 \pm 40.6 \mu \mathrm{g} / \mathrm{ml})$ and P-Ch C $(56.4 \pm 32.3$ $\mu \mathrm{g} / \mathrm{ml})$ individuals, as compared to the control group $(135.9 \pm 43.6 \mu \mathrm{g} / \mathrm{ml})$; p-value was $<0.01$ and $<0.001$, respectively. Moreover, a significant difference in the concentration of afamin was observed between patients with P-Ch stage C and P-Ch stage A of disease $(124.8 \pm 72.4$ $\mu \mathrm{g} / \mathrm{ml}$ ); p-value was $<0.01$ ) (Figure 1A). 
As far as adropin is concerned, a reverse relationship was demonstrated: the highest concentration was found in patients with P-Ch C $(11.7 \pm 5.7 \mathrm{ng} / \mathrm{ml})$ cirrhosis. Furthermore, the above concentration was significantly higher compared to patients with P-Ch A cirrhosis $(7.2 \pm 2.8 \mathrm{ng} / \mathrm{ml} ; \mathrm{p}<0.05)$ and controls $(7.5 \pm 2.6 \mathrm{ng} / \mathrm{ml} ; \mathrm{p}<0.05)$ (Figure 1B).

To evaluate the independent effects of various variables on the concentration of afamin and adropin, a multiple linear regression analysis was performed. The following variables were used: stage of disease according to the P-Ch classification and the additional laboratory parameters outside this classification. The factors independently associated with serum concentrations of afamin were found to be: stage of liver cirrhosis according to the PCh score (the highest relative effect on the variability of afamin concentration), activity of ASP and concentration of urea. This model accounted only for $15 \%$ of variability of an independent variable albeit was optimal under given conditions (Table 4).

In the case of adropin, the regression model contained only two independent variables: stage of cirrhosis according to the P-Ch score and concentration of CRP. The model accounted for $17 \%$ of variability of serum adropin concentration and the highest relative effect on this variability was attributable to the stage of disease according to the P-Ch score (Table 5).

Receiver-operators characteristic (ROC) curves used for diagnostic accuracy of afamin and adropin are given in Figure 2.

\section{Discussion}

In our study, the plasma concentration of afamin was found to decrease with the degree of severity of alcoholic liver cirrhosis. Afamin is a protein belonging to the albumin family encompassing albumin, alpha-fetoprotein and vitamin D-binding protein. Afamin is a glycoprotein with a molecular weight of $87 \mathrm{kDa}$, whose $55 \%$ of amino acid sequences are 
similar to an albumin and which acts as a protein transporting vitamin $\mathrm{E}$ in plasma ${ }^{\mathrm{ix}}$. Afamin circulating in plasma is primarily produced in the liver ${ }^{\mathrm{x}}$.

Recent studies have proven that afamin is involved in the development of metabolic disorders. In the study involving $>20000$ patients, a strong correlation, independent of other metabolic risk factors, has been demonstrated between the concentration of afamin versus the concentration of insulin and type 2 diabetes mellitus ${ }^{\mathrm{xi}}$.

The studies in patients with ovarian cancer and stomach cancer have demonstrated significantly reduced levels of afamin ${ }^{x i i}$. Moreover, the concentration of afamin was found to be significantly decreased in patients with sepsis and pneumonia ${ }^{\text {xiii }}$. Otherwise, no changes in concentrations of afamin were observed in patients with chronic obstructive pulmonary disease (COPD) and chronic renal disease. In cases of heart failure, concentrations of afamin were only slightly reduced [Dieplinger 2015]. According to literature data, the concentration of afamin has been reversely correlated with the concentration of C-reactive protein (CRP) and of interleukin- $6^{\mathrm{xiv}}$. Therefore, afamin is considered a negative acute phase protein.

To the best of our knowledge, our study is the first to focus on determinations of plasma concentrations of afamin in patients with alcoholic liver cirrhosis. Our findings demonstrated reduced serum concentrations of afamin in patients with alcoholic liver cirrhosis. The higher the stage of the disease, the lower the concentrations of afamin. However, contrary to the other groups of patients, CRP was not found to be an independent predictor of afamin concentrations. In the multi-factorial model, the variability of afamin concentrations was most strongly affected by the Pugh-Child category, which can be explained by impaired anabolic function of the liver during cirrhosis. The above is analogical to hypoalbuminaemia observed in cirrhosis-related renal failure when concentrations of albumins are reduced, their structure is impaired and they are excessively intravascularly 
damaged due to increased inflammatory and pro-oxidative processes, which characterise advanced liver cirrhosis ${ }^{\mathrm{xv}}$.

The second protein analysed in our study was adropin. Adropin is a factor involved in regulation of homeostasis of carbohydrates and lipids and is associated with energy homeostasis of the body ${ }^{\mathrm{xvi}}$. Adropin protects against fatty liver and obesity-related hyperinsulinaemia and is a factor regulating lipogenesis - affecting the gene expression in the liver and peroxisome proliferator-acivated receptor gamma in the adipose tissue [Kumar 2008].

Adropin is produced not only in the liver but also in the pancreas and brain ${ }^{\text {xvii }}$ [Kumar 2008]. Its concentration can change in various pathological conditions. The available data regarding the role of adropin and relationships with the development with metabolic diseases, are inconsistent. Reduced levels of adropin have been observed in obesity-associated insulin resistance, gestational diabetes, nonalcoholic fatty liver disease (NAFLD), acute myocardial infarction and endothelial dysfunction [Marczuk]. Sayin et al. have demonstrated that low adropin concentrations were associated with the risk of NAFLD ${ }^{\text {xviii }}$. It should be stressed, however, that their study involved children and not adults. However, existing data are conflicting. Increased adropin concentrations have been found in patients with heart failure, type 2 diabetes and diabetic nephropathy ${ }^{\mathrm{xix}},{ }^{\mathrm{xx}}$.

The available literature lacks studies on the role of adropin in liver cirrhosis. In our study, the concentration of adropin was positively correlated with the stage of liver cirrhosis according to the Pugh-Child score. Additionally, in the multifactorial model, an independent factor associated with the level of adropin was C-reactive protein. Nevertheless, the model designed accounted only for $17 \%$ of variability of the dependent variable and thus cannot be used for prognostic purposes. 
Considering the complex nature of metabolic disorders in compensated liver cirrhosis, it cannot be excluded that elevated adropin concentrations noted in our study resulted from feedback mechanisms leading to increased extra-hepatic synthesis.

Receiver-operator characteristic (ROC) curves showed that adropin is not a valuable diagnostic marker for alcoholic cirrhosis. On the other hand, afamin is characterized by relatively high specificity but low sensitivity (Figure 2). It may result in false positive results therefore its clinical utility requires further investigations.

Our study had several limitations. Firstly, this was a single-centre study and the sample size was insufficient to come to final conclusions. Therefore, further prospective studies in a large population are needed. Moreover, it should be emphasised that multifactorial models accounted only for a small part of variability $(<20 \%)$ of plasma concentrations of afamin and adropin; therefore, some other factors potentially affecting their concentrations should be search for. Our study did not involve patients with other than alcoholic liver cirrhosis; thus, it cannot be determined whether the observed relationships also regard some other group of patients.

\section{Conclusions}

The concentration of afamin decreases with the severity of alcoholic liver cirrhosis, which most likely results from impaired hepatic synthesis. Otherwise, the higher the stage of disease according to the Child-Pugh score, the higher the concentration of adropin. Further prospective studies are required to explain the role of both these proteins in alcoholic liver cirrhosis.

\section{Acknowledgments}


Authors thank Anna Misiuna M.A., who provided medical writing services on behalf of Medical University of Lublin, Poland.

\section{Conflicts of Interest}

The authors declare no conflicts of interest.

\section{TABLES \& FIGURES}

Table 1. Demographic and clinical characteristics of study and control groups.

\begin{tabular}{|c|c|c|c|c|}
\hline & \multirow{2}{*}{$\begin{array}{l}\text { Control } \\
\text { group } \\
(n=20)\end{array}$} & \multicolumn{3}{|c|}{ Alcoholic liver cirrhosis $(n=99)$} \\
\hline & & $\begin{array}{l}\text { P-Ch A } \\
(n=29)\end{array}$ & $\begin{array}{l}\text { P-Ch B } \\
(n=36)\end{array}$ & $\begin{array}{l}\text { P-Ch C } \\
(n=34)\end{array}$ \\
\hline Age (years) & $48.9 \pm 15.1$ & $53.3 \pm 12.3$ & $54.6 \pm 11.0$ & $56.9 \pm 7.7$ \\
\hline The percentage of men & $65 \%$ & $72.4 \%$ & $66.7 \%$ & $58.8 \%$ \\
\hline Height $(\mathrm{cm})$ & $170.4 \pm 6.6$ & $171.8 \pm 7.8$ & $175.3 \pm 8.4$ & $173.4 \pm 6.9$ \\
\hline Body mass $(\mathrm{kg})$ & $67.8 \pm 7.8$ & $68.1 \pm 14.8$ & $71.5 \pm 13.2$ & $70.2 \pm 12.8$ \\
\hline $\begin{array}{l}\text { Time of alcohol abuse } \\
\text { (years) }\end{array}$ & - & $13.1 \pm 4.8$ & $14.1 \pm 4.9$ & $15.7 \pm 5.4$ \\
\hline Ascites (\%) & 0 & $32 \%$ & $61 \%$ & $85 \%$ \\
\hline Oesophageal varices (\%) & 0 & $14 \%$ & $45 \%$ & $88 \%$ \\
\hline Encephalopathy (\%) & 0 & $28 \%$ & $151 \%$ & $91 \%$ \\
\hline
\end{tabular}


Table 2. Biochemical characteristics of study and control groups.

\begin{tabular}{|c|c|c|c|c|}
\hline & \multirow{2}{*}{$\begin{array}{l}\text { Control } \\
\text { group } \\
(n=20)\end{array}$} & \multicolumn{3}{|c|}{ Alcoholic liver cirrhosis $(n=99)$} \\
\hline & & $\begin{array}{l}\text { P-Ch A } \\
(n=29)\end{array}$ & $\begin{array}{l}\text { P-Ch B } \\
(n=36)\end{array}$ & $\begin{array}{l}\text { P-Ch C } \\
(n=34)\end{array}$ \\
\hline Bilirubin (mg/dl) & $0.68 \pm 0.28$ & $2.21 \pm 1.4$ & $4.12 \pm 3.25$ & $7.89 \pm 7.94$ \\
\hline Albumin $(\mathrm{g} / \mathrm{dl})$ & - & $3.27 \pm 0.76$ & $2.78 \pm 0.6$ & $2.44 \pm 0.46$ \\
\hline $\begin{array}{l}\text { International } \\
\text { normalized ratio, INR }\end{array}$ & - & $1.25 \pm 0.27$ & $1.44 \pm 0.29$ & $1.68 \pm 0.41$ \\
\hline Platelets (G/1) & $226.8 \pm 35.8$ & $186.03 \pm 76.9$ & $123.6 \pm 66.25$ & $114.11 \pm 61.6$ \\
\hline $\begin{array}{l}\text { Mean cell volume, } \\
\mathrm{MCV}(\mathrm{fl})\end{array}$ & $94.65 \pm 4.45$ & $92.38 \pm 6.25$ & $91.9 \pm 10.08$ & $97.53 \pm 8.02$ \\
\hline Urea (mg/dl) & - & $32.04 \pm 20.1$ & $23.49 \pm 15.62$ & $39.58 \pm 16.1$ \\
\hline Sodium (mmol/1) & $139.82 \pm 3.24$ & $133.67 \pm 5.3$ & $135.38 \pm 3.6$ & $133.51 \pm 6.63$ \\
\hline Potassium (mmol/l) & $4.41 \pm 0.37$ & $3.88 \pm 0.6$ & $3.94 \pm 0.6$ & $3.3 \pm 0.66$ \\
\hline $\begin{array}{l}\text { C-reactive protein, } \\
\text { CRP (mg/l) }\end{array}$ & $2.11 \pm 1.96$ & $14.97 \pm 12.62$ & $19.21 \pm 17.35$ & $20.8 \pm 19.92$ \\
\hline $\begin{array}{l}\text { Aspartate transaminase, } \\
\text { ASP (U/l) }\end{array}$ & $18.1 \pm 5.2$ & $53.1 \pm 21.8$ & $145.5 \pm 110.7$ & $188.3 \pm 107.4$ \\
\hline
\end{tabular}


Table 3. Afamin and adropin in cirrhotic patients and controls.

\begin{tabular}{|c|c|c|c|c|c|}
\hline & \multirow{2}{*}{$\begin{array}{l}\text { Control } \\
\text { group } \\
(n=20)\end{array}$} & \multicolumn{3}{|c|}{ Alcoholic liver cirrhosis $(n=99)$} & \multirow[b]{2}{*}{$\mathbf{p}$} \\
\hline & & $\begin{array}{l}\text { P-Ch A } \\
(n=29)\end{array}$ & $\begin{array}{l}\text { P-Ch B } \\
(n=36)\end{array}$ & $\begin{array}{l}\text { P-Ch C } \\
(n=34)\end{array}$ & \\
\hline $\begin{array}{l}\text { Afamin } \\
(\mu \mathrm{g} / \mathrm{ml})\end{array}$ & $135.9 \pm 43.6$ & $124.8 \pm 72.4$ & $85.1 \pm 40.6$ & $56.4 \pm 32.3$ & $<0.0001$ \\
\hline $\begin{array}{l}\text { Adropin } \\
(\mathrm{ng} / \mathrm{ml})\end{array}$ & $7.5 \pm 2.6$ & $7.2 \pm 2.8$ & $8.4 \pm 43.6$ & $11.7 \pm 5.7$ & 0.02 \\
\hline
\end{tabular}


Table 4. Factors influencing afamin concentration - multiple linear regression analysis

\begin{tabular}{|l|l|l|l|l|l|}
\hline Independent & $\mathbf{b}^{*}$ & SE & $\mathbf{b}$ & SE & $\mathbf{p}$ \\
\hline intercept & & & & & \\
\hline P-Ch & -0.27 & 0.07 & -10.4 & 2.9 & $<0.00001$ \\
category & & & & & \\
\hline Urea & -0.2 & 0.08 & -0.4 & 0.17 & 0.01 \\
\hline ASP & -0.16 & 0.07 & -0.06 & 0.03 & 0.04 \\
\hline Model: $\mathrm{R}^{2}=0.18$, adjusted $\mathrm{R}^{2}=0.15, \mathrm{p}<0.0001$. & & \\
\hline
\end{tabular}

$\mathrm{b}^{*}$ - standardized coefficient of regression, $\mathrm{b}$ - coefficient of regression, SE - standard error, $\mathrm{R}^{2}-$ coefficient of determination. 
Table 5. Factors influencing adropin concentration - multiple linear regression analysis.

\begin{tabular}{|l|l|l|l|l|l|}
\hline $\begin{array}{l}\text { Independent } \\
\text { variables }\end{array}$ & $\mathbf{b}^{*}$ & SE & $\mathbf{b}$ & SE & $\mathbf{p}$ \\
\hline intercept & & & 6.04 & 1.03 & $<0.00001$ \\
\hline P-Ch & 0.33 & 0.14 & 0.04 & 0.018 & 0.02 \\
\hline CRP & 0.3 & & & 0.5 & 0.04 \\
\hline Model: $\mathrm{R}^{2}=0.21$, adjusted $\mathrm{R}^{2}=0,17, \mathrm{p}<0,009$. & & & \\
\hline
\end{tabular}

b*- standardized coefficient of regression, $b$ - coefficient of regression, SE - standard error, $\mathrm{R}^{2}-$ coefficient of determination. 
Figure 1. Afamin (A) and adropin (B) concentrations in patients with liver cirrhosis and controls.
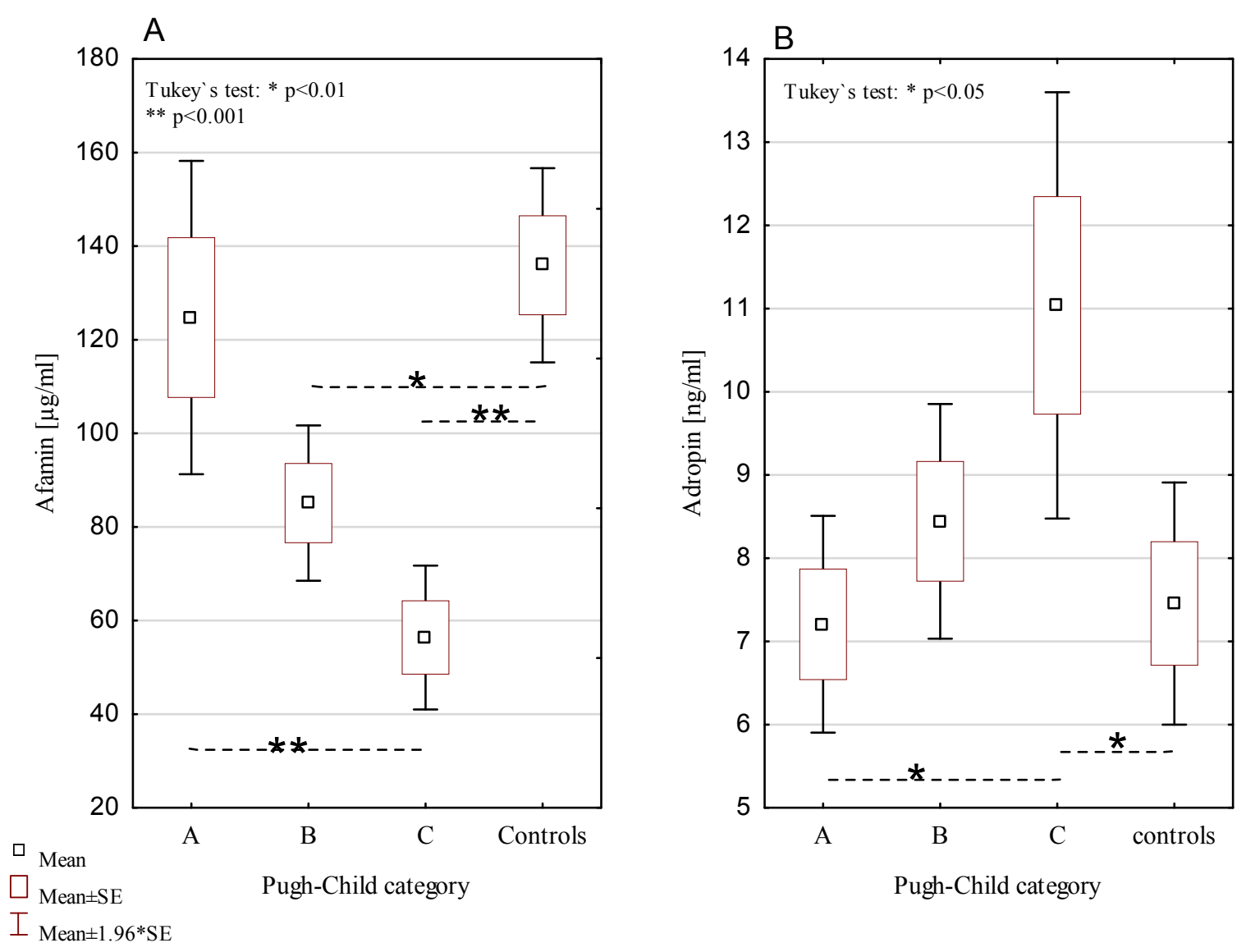
Figure 2. Receiver-operator characteristic (ROC) curves for diagnostic accuracy of afamin and adropin.

Adropin and liver cirrhosis diagnosis (P-Ch A-C)

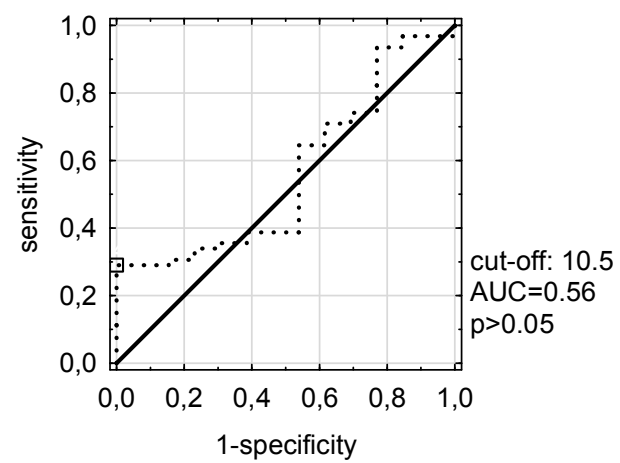

Afamin and decompensated liver cirrhosis diagnosis (P-Ch C)

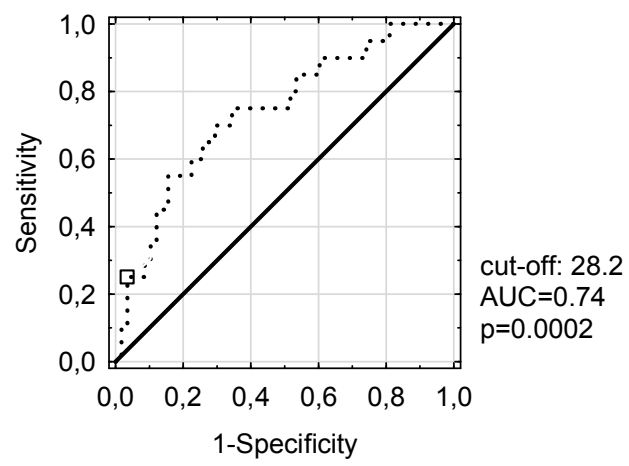

Adropin and decompensated liver cirrhosis diagnosis (P-Ch C)

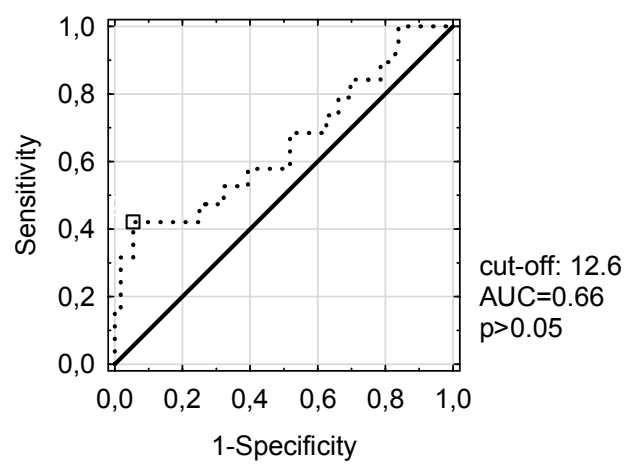

Afamin and liver cirrhosis diagnosis (P-Ch A-C)

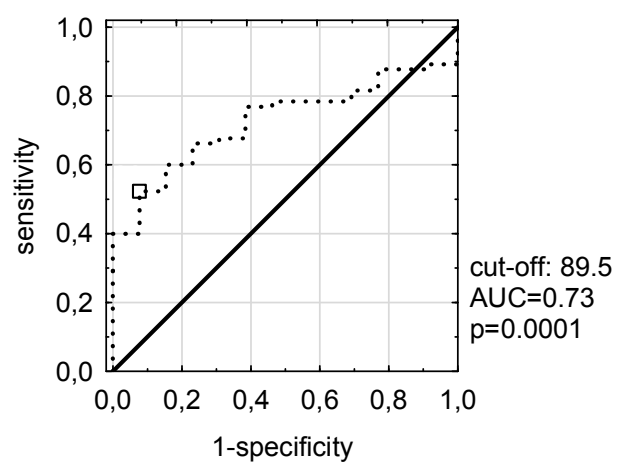




\section{References}

${ }^{i}$ Mokdad A, Lopez A, Shahraz S, et al. Liver cirrhosis mortality in 187 countries between 1980 and 2010: a systematic analysis. BMC Medicine 2014; 12: 145.

ii Seth, D.; D’Souza El-Guindy, N.B.; Apte, M.; Mari, M.; Dooley, S.; Neuman, M.; Haber, P.S. Alcohol, signaling, and ECM turnover. Alcohol Clin. Exp. Res. 2010, 34, 4-18.

iii Zhou W-C, Zhang Q-B, Qiao L. Pathogenesis of liver cirrhosis. World J Gastroenterol. 2014; 20(23): 7312-7324.

iv Ishii H, Horie Y, Yamagshi Y, Ebinuma H. Alcoholic Liver Disease and Its Relationship with Metabolic Syndrome. JMAJ 53(4): 236-242, 2010.

${ }^{\text {vv }}$ Seeber B, Morandell E, Lunger F, Wildt L, Dieplinger H. Afamin serum concentrations are associated with insulin resistance and metabolic syndrome in polycystic ovary syndrome. Reproductive Biology and Endocrinology 2014, 12:88

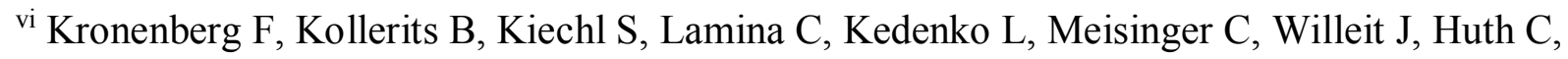
Wietzorrek G, Altmann ME, Thorand B, Melmer A, Dähnhardt D, Santer P, Rathmann W, Paulweber B, Koenig W, Peters A, Adham IM, Dieplinger H: Plasma concentrations of afamin are associated with the prevalence and development of metabolic syndrome. Circ Cardiovasc Genet 2014, doi:10.1161/CIRCGENETICS.113.000654.

vii Marczuk N, Cecerska-Heryć E, Jesionowska A, Dołęgowska B. Adropin-physiological and pathophysiological role. Postepy Hig Med Dosw (online), 2016; 70: 981-988

${ }^{\text {viii }}$ Pugh R, Murray-Lyon I, Dawson J, Pietroni M et al. Transection of the oesophagus for bleeding oesophageal varices. Br J Surg. 1973, 60(8): 646-9. 
${ }^{\text {ix }}$ H.S. Lichenstein, D.E. Lyons, M.M. Wurfel, et al.Afamin is a new member of the albumin, a-fetoprotein, and vitamin D-binding protein gene family. J Biol Chem, 269 (1994), pp. 18149-18154

${ }^{x}$ Dieplinger H, Dieplinger B. Afamin--A pleiotropic glycoprotein involved in various disease states. Clin Chim Acta. 2015 Jun 15;446:105-10. doi: 10.1016/j.cca.2015.04.010.

${ }^{x i}$ Kollerits B, Lamina C, Huth C, Marques-Vidal P, Kiechl S, Seppälä I, Cooper J, Hunt S, Meisinger C, Herder $\mathrm{C}$ et al. Plasma Concentrations of Afamin Are Associated With Prevalent and Incident Type 2 Diabetes: A Pooled Analysis in More Than 20,000 Individuals. Diabetes Care 2017 Aug; dc170201. https://doi.org/10.2337/dc17-0201

${ }^{\text {xii }}$ H. Dieplinger, D.P. Ankerst, A. Burges, et al.Afamin and apolipoprotein A-IV: novel protein markers for ovarian cancer. Cancer Epidemiol Biomarkers Prev, 18 (2009), pp. 1127 1133

xiii Dieplinger B, Egger M, Gabriel C, Poelz W, Morandell E, Seeber B, Kronenberg F, Haltmayer M, Mueller T, Dieplinger H. Analytical characterization and clinical evaluation of an enzyme-linked immunosorbent assay for measurement of afamin in human plasma. Clin Chim Acta. 2013 Oct 21;425:236-41. doi: 10.1016/j.cca.2013.08.016.

xiv B. Dieplinger, M. Egger, C. Gabriel, et al. Analytical characterization and clinical evaluation of an enzyme-linked immunosorbent assay for measurement of afamin in human plasma. Clin Chim Acta, 425 (2013), pp. 236-241

${ }^{\mathrm{x}}$ Lee JS. Albumin for End-Stage Liver Disease. The Korean Journal of Internal Medicine. 2012;27(1):13-19. doi:10.3904/kjim.2012.27.1.13.

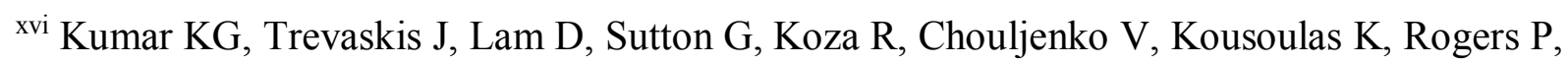
Kesterson R, Thearle $\mathrm{M}$ et al. Identification of Adropin as a Secreted Factor Linking Dietary Macronutrient Intake with Energy Homeostasis and Lipid Metabolism. Cell Metabolism 8, 468-481, December 3, 2008.

${ }^{x v i i}$ Aydin S. Three new players in energy regulation: preptin, adropin and irisin. Peptides. 2014 Jun;56:94-110. doi: 10.1016/j.peptides.2014.03.021.

xviii Sayın, O., Tokgöz, Y. \& Arslan, N. (2014). Investigation of adropin and leptin levels in pediatric obesity-related nonalcoholic fatty liver disease. Journal of Pediatric Endocrinology and Metabolism, 27(5-6), pp. 479-484.doi:10.1515/jpem-2013-0296

${ }^{\text {xix }}$ Lian W, Gu X, Qin Y, Zheng X. Elevated Plasma Levels of Adropin in Heart Failure Patients. Intern Med 50: 1523-1527, 2011 DOI: 10.2169/internalmedicine.50.5163 
${ }^{\mathrm{xx}} \mathrm{Hu} \mathrm{W}$, Chen L. Association of Serum Adropin Concentrations with Diabetic Nephropathy. Mediators of Inflammation. Volume 2016, Article ID 6038261, 5 pages. http://dx.doi.org/10.1155/2016/6038261 